\title{
Shear Viscosity from the Effective Coupling of Gravitons
}

\author{
Rong-Gen Cai, Zhang-Yu Nie and Ya-Wen Sun \\ Institute of Theoretical Physics, Chinese Academy of Sciences, \\ Beijing 100190, China \\ E-mail: cairg,niezy,sunyw@itp.ac.cn
}

\begin{abstract}
We review the progress in the holographic calculation of shear viscosity for strongly coupled field theories. We focus on the calculation of shear viscosity from the effective coupling of transverse gravitons and present some explicit examples.
\end{abstract}

Keywords: Shear viscosity; AdS/CFT.

\section{Introduction}

The AdS/CFT correspondence ${ }^{1-4}$ provides a new way to solve strongly coupled field theory problems. Especially, we can calculate the transport coefficients in the hydrodynamic limit of the field theory from the gravity side. Shear viscosity is one of these coefficients that have been vastly studied through the holographic method. ${ }^{5-8}$ In the early days, the shear viscosity was calculated in a lot of theories dual to Einstein gravity with or without chemical potential. ${ }^{9-12}$ It was found that the value of the shear viscosity over the entropy density ratio $\eta / s$ is always $1 / 4 \pi$. After considering large $\mathrm{N}$ effects, this ratio gets positive corrections. Then it was conjectured that there is a universal lower bound on the ratio $\eta / s$ in nature $\left(\mathrm{KSS}\right.$ bound $\left.^{8}\right)$. Known experiments such as the Quark-Gluon Plasma(QGP) in RHIC also satisfy this conjecture. Then more attention has been payed to the study of the universality of $\eta / s$ through holographic method, and the $\eta / s$ was found to be still above $1 / 4 \pi$ in Ref. 13-20. However, it was later found that the KSS bound can be slightly violated in theories with gravity dual of more general theories of gravity. ${ }^{21}$

In the study of the $\eta / s$ through holographic method, it was gradually found that the shear viscosity of the boundary field theory only depends on the effective coupling of the transverse gravitons valued on the horizon in the gravity side. ${ }^{22-26}$ This relation applies to systems dual to more general 
theories beyond Einstein gravity, and may shed some light on the understanding of the AdS/CFT correspondence in another point of view. We will shortly review the progress of studies on this problem in this article, and also introduce some interesting results using the language of the effective coupling.

The plan of this paper is as follows. In the next section, we review the progress in the study of shear viscosity using holographic method. In Sec. 3 , we introduce recent studies on the calculation of shear viscosity from the effective coupling of transverse gravitons, and give the result in AdS Gauss-Bonnet gravity. In Sec. 4 we present some examples from the effective coupling.

\section{Shear viscosity from AdS/CFT}

The AdS/CFT correspondence ${ }^{1-4}$ is a duality between the anti-de Sitter (AdS) spacetime and a conformal field theory (CFT) living on the boundary of the AdS spacetime. In details, suppose that we have a field $\phi$ moving in the AdS spacetime and a corresponding operator $\mathcal{O}$ in the CFT side, and the boundary value $\phi_{0}$ of $\phi$ coupled to the boundary operator $\mathcal{O}$ as a source, then the partition function of $\phi$ with boundary value $\phi_{0}$ in the gravity side is equal to the generating functional of $\mathcal{O}$ with a source $\phi_{0}$ in the CFT side

$$
Z\left(\phi_{0}\right)=\left\langle e^{i \int \phi_{0} \mathcal{O}}\right\rangle_{C F T}
$$

In order to get some information in the CFT, we only need to study the partition function $Z\left(\phi_{0}\right)$ in the gravity side. In the AdS/CFT correspondence, the gravity system in the AdS side is classical at low energies, so the partition function $Z\left(\phi_{0}\right)$ can be just determined by the classical solution of the field $\phi$

$$
Z\left(\phi_{0}\right)=e^{i S(\phi)}
$$

where $S(\phi)$ is the classical action of the classical solution $\phi$ with boundary value $\phi_{0}$. Then with this correspondence, we can easily get the correlation functions of the operator $\mathcal{O}$ in the strongly coupled field theory by the standard functional formulas.

In the point of view of the field theory, knowing the correlation functions of operators, one can extract transport coefficients using the Kubo formula. Applying this formula to the case of energy momentum tensor, we can calculate the shear viscosity by ${ }^{27,28}$

$$
\eta=\lim _{\omega \rightarrow 0} \frac{1}{2 \omega i}\left(G_{x y, x y}^{A}(\omega, 0)-G_{x y, x y}^{R}(\omega, 0)\right),
$$


where $\eta$ is the shear viscosity, and the retarded Green's function is defined by

$$
G_{\mu \nu, \lambda \rho}^{R}(k)=-i \int d^{4} x e^{-i k \cdot x} \theta(t)\left\langle\left[T_{\mu \nu}(x), T_{\lambda \rho}(0)\right]\right\rangle .
$$

The advanced Green's function can be related to the retarded Green's function by $G_{\mu \nu, \lambda \rho}^{A}(k)=G_{\mu \nu, \lambda \rho}^{R}(k)^{*}$.

Using the AdS/CFT duality and the Kubo formula, the shear viscosity $\eta$ can be calculated from the gravity dual, and interesting results have been obtained using this holographic method. It was found that the ratio of shear viscosity over entropy $\eta / s$ calculated from gravity dual of Einstein gravity is always $1 / 4 \pi$. After considering some corrections from large $\mathrm{N}$ effect, the ratio gets positive corrections. Notice that in all the known experiments, the fluids measured all have a ratio $\eta / s$ larger than $1 / 4 \pi$, The authors of Ref. 8 conjectured that this ratio may have a universal lower bound in real fluids in nature, called the KSS bound.

\section{Shear viscosity and the effective coupling of transverse gravitons}

It has recently been conjectured that the shear viscosity depends on the effective coupling of transverse gravitons valued on the horizon. ${ }^{23} \mathrm{In}$ Ref. $22-24$ the authors conjectured that this ratio is equal to a quotient of effective couplings of two different polarizations of gravitons, $\kappa_{x y}$ and $\kappa_{r t}$ valued on the horizon. Then the dependence of shear viscosity on the effective coupling of transverse gravitons is confirmed in Ref. 25 using a membrane paradigm and in Ref. 26 by calculating the on-shell action of the transverse gravitons. In Sec. 3.1 we show the relation of the shear viscosity and the effective coupling of transverse gravitons. In Sec. 3.2 we discuss the case of Gauss-Bonnet gravity.

\subsection{Transverse Graviton}

For simplicity, we consider a five dimensional asymptotic AdS black hole solution with a Ricci flat horizon to some kind of gravity theory with or without matter fields. We write the metric as

$$
d s^{2}=-g(u)(1-u) d t^{2}+\frac{1}{h(u)(1-u)} d u^{2}+\frac{r_{h}^{2}}{u l}\left(d x^{2}+d y^{2}+d z^{2}\right),
$$

where $r_{h}$ is the horizon radius and $u=r_{h} / r$. Then the horizon is located at $u=1$ and the boundary lives at $u=0 . g(u)$ and $h(u)$ are two functions 
regular at $u=1$ and $l$ is the AdS radius which is related to the cosmological constant by $\Lambda=-6 / l^{2}$.

The boundary of this AdS spacetime is a $\mathbb{R}^{4}$, on which a CFT of some kind lives. To calculate the shear viscosity of this CFT, we need the Green functions of the energy momentum tensor, which we can get by making a small perturbation to the metric tensor in the gravity side. For simplicity, we choose spatial coordinates $x, y, z$, so that the momentum of the perturbations points along the z-axis. Then the perturbations can be written as $h_{\mu \nu}=h_{\mu \nu}(t, z, u)$. In this basis there are three groups of gravity perturbations, each of which is decoupled from others: the scalar, vector and tensor perturbations. ${ }^{29}$ Here we use the simplest one that can be used to calculate the shear viscosity, the tensor perturbation $h_{x y}$. We use $\phi$ to denote this perturbation $\phi=h_{y}^{x}$ and write $\phi$ in a basis as $\phi(t, u, z)=\phi(u) e^{-i \omega t+i p z}$. Then by expanding the gravity action to the second order with the background metric (5), one can get the effective action of the transverse graviton

$$
S=\frac{1}{16 \pi G} \int d^{5} x \sqrt{-g} K_{e f f}(u)\left(\nabla_{\mu} \phi \nabla^{\mu} \phi\right)
$$

where we assume the graviton to be a minimal coupled massless scalar with an effective coupling $K_{\text {eff }}$. Then it can be proved that the shear viscosity depends only on the effective coupling valued on the horizon $K_{\text {eff }}(u=$ 1). ${ }^{25,26}$

In Ref. 25, the authors used the membrane paradigm to analysis the universality of shear viscosity over entropy ratio, and proved that the shear viscosity depends on the coupling of the transverse gravitons valued on the horizon. This shows some relations between the fluid on the near horizon slicing and the long wavelength low frequency limit of the boundary CFT. The boundary theory can be seen as flowing from the horizon physics throw some spacetime structures.

In the paper Ref. 26 , we directly calculated the on-shell action of the transverse gravitons, and obtained the shear viscosity expressed by the effective coupling of transverse gravitons. In Einstein gravity, this effective coupling is always $-1 / 2$, so the shear viscosity over entropy density is always $1 / 4 \pi$ in theories with Einstein gravity dual. But in modified gravity theories, the effective coupling of transverse gravitons could be different. For example, in gravity theory with $R^{2}$ corrections, the effective coupling of transverse gravitons gets corrections, thus in the dual field theory, the universal low bound $1 / 4 \pi$ can be violated. ${ }^{21,30-33}$ 


\subsection{KSS bound violation in Gauss-Bonnet gravity}

The $R^{2}$ corrections come from string theory or some other UV completion of Einstein gravity. After field redefinitions these theories reduce to GaussBonnet gravity up to the $R^{2}$ order. The action of Gauss-Bonnet gravity is

$$
S=\frac{1}{16 \pi G} \int d^{5} x \sqrt{-g}\left(R-2 \Lambda+\frac{\lambda l^{2}}{2} R_{G B}\right)
$$

in which

$$
R_{G B}=\left(R^{2}-4 R^{\mu \nu} R_{\mu \nu}+R^{\mu \nu \rho \sigma} R_{\mu \nu \rho \sigma}\right)
$$

is the Gauss-Bonnet term, $\lambda$ is the Gauss-Bonnet parameter with a small positive value.

With the Gauss-Bonnet term, the action of the transverse gravitons is still that of minimal coupled massless scalar, but the effective coupling differs from the value in pure Einstein gravity(it is no longer a constant and depends on the radial coordinate $u$ ). Thus the ratio of shear viscosity over entropy density $\eta / s$ in the dual field theory also differs from the universal value with Einstein gravity dual. The ratio in the Gauss-Bonnet case is calculated to be

$$
\frac{\eta}{s}=\frac{1}{4 \pi}(1-4 \lambda)
$$

With a positive Gauss-Bonnet correction $\lambda>0$, the universal lower bound of $1 / 4 \pi$ will obviously be violated. But with causality consideration from the CFT side, the Gauss-Bonnet parameter $\lambda$ is constrained as $\lambda<0.09,{ }^{30}$ and then the ratio $\eta / s$ will have a new lower bound as

$$
\frac{\eta}{s} \geq \frac{4}{25 \pi} \text {. }
$$

The calculation of $\eta / s$ from the effective coupling of the transverse gravitons is convenient, and we will give more examples of studying the ratio $\eta / s$ with this method. More discussions on the shear viscosity from the effective coupling of transverse gravitons could be found in Ref. 34-40.

\section{More examples from effective coupling}

Since the violation of the KSS bound was discovered, the universality of the bound of $\eta / s$ needs more attention, it would be valuable to examine wether the ratio will get more corrections in more general gravity duals.

In our previous work, ${ }^{26,41}$ we calculated the shear viscosity in the case of AdS Gauss-Bonnet gravity with $F^{4}$ term corrections of Maxwell field, 
and in AdS Gauss-Bonnet gravity with dilaton coupling. In both the two cases, the ratio of shear viscosity over entropy density gets more corrections from the AdS Gauss-Bonnet case. We also considered the shear viscosity in the extremal case in a recent work. ${ }^{42}$ We give our results here.

\subsection{Gauss-Bonnet Gravity with $F^{4}$ term corrections of Maxwell field}

In Ref. 43, when Maxwell field is added to the Gauss-Bonnet gravity, the ratio $\eta / s$ gets positive corrections to the pure AdS Gauss-Bonnet gravity case. Then in our work, ${ }^{26}$ we further studied the effect of non-linear term of Maxwell field on the shear viscosity in the setup of Gauss-Bonnet gravity dual. We found that the $F^{4}$ term has some effects on the ratio $\eta / s$. We further found that with Maxwell field, with or without $F^{4}$ terms, the ratio $\eta / s$ depends on the temperature $T$ in the way

$$
\frac{\eta}{s}=\frac{1}{4 \pi}\left(1-\frac{4 \lambda \pi l^{2}}{r_{h}} T\right) .
$$

This is very interesting, for that the effect of the Maxwell field on $\eta / s$ is simply relating this ratio to the temperature of the black hole.

\subsection{AdS Gauss-Bonnet gravity with dilaton coupling}

The dilaton field coupled to the Gauss-Bonnet gravity has non-trivial contributions, for that the pure Gauss-Bonnet black hole solution without the dilaton is not a solution of the case with dilaton by simply requiring the dilaton being a constant. So it would be interesting to see whether the dilaton field has contributions to the shear viscosity in the dual field theory. In Ref. 41, we found that in the case of Gauss-Bonnet gravity with dilaton coupling the effective action of the transverse gravitons is still the type of minimally coupled massless scalar, so the shear viscosity depends on the effective coupling of the transverse gravitons. We calculated the ratio of shear viscosity over entropy density, and the result is

$$
\frac{\eta}{s}=\frac{1}{4 \pi}\left(1-4 \lambda e^{(\tau-\gamma) \phi_{d}(1)}\left(1-24 \gamma^{2} \lambda e^{(\tau-\gamma) \phi_{d}(1)}+3 \gamma \tau\right)\right) .
$$

After considering the causality restriction, we can see that with dilaton coupling, the new lower bound in AdS Gauss-Bonnet case of the $\eta / s$ can be further violated in the parameter space of the black hole solution. 


\subsection{Shear viscosity at zero temperature}

It is well known that the extremal black hole is very different from the ordinary black holes with a finite temperature. In a recent paper, ${ }^{44}$ shear viscosity and other transport coefficients of field theories with nonzero chemical potentials were calculated at zero temperature in the background of extremal AdS RN black holes using similar methods as in Ref. 45 where properties of non-Fermi fluid ${ }^{46-48}$ are discussed from AdS/CFT, there the IR physics plays an important role in deriving the UV physics on the boundary. In the extremal case of Einstein gravity, the shear viscosity over entropy density is still $1 / 4 \pi$.

We calculated the shear viscosity in field theories dual to Gauss-Bonnet gravity at zero temperature with non-zero chemical potential. We confirmed that in the extremal case, the shear viscosity also depends on the effective coupling of transverse gravitons valued on horizon. Our results show that, the shear viscosity over entropy density in field theory dual to Gauss-Bonnet gravity at zero temperature is still $1 / 4 \pi$, the same as that in Einstein gravity.

\section{Conclusion}

In this paper we gave a brief review on the progress of calculating the shear viscosity in strongly coupled field theory through holographic method, with special attention to the connection between the shear viscosity and the effective coupling of transverse gravitons in the dual gravity side. This connection is applicable for a wide class of gravity theories. We presented some examples of this connection in Gauss-Bonnet gravity with Maxwell field, and the case with dilaton coupling. We also discussed results with extremal black holes.

\section{Acknowledgments}

We would like to thank N. Ohta and Yan Liu for useful discussions and collaborations in some relevant studies. RGC thanks the organizers ICGA9 for a warm hospitality during the conference. This work was supported partially by grants from NSFC, China (No. 10535060, No. 10821504 and No. 10975168) and a grant from MSTC, China (No. 2010CB833004).

\section{References}

1. J. M. Maldacena, "The large N limit of superconformal field theories and supergravity," Adv. Theor. Math. Phys. 2, 231 (1998) [Int. J. Theor. Phys. 
38, 1113 (1999)] [arXiv:hep-th/9711200].

2. S. S. Gubser, I. R. Klebanov and A. M. Polyakov, "Gauge theory correlators from non-critical string theory," Phys. Lett. B 428, 105 (1998) [arXiv:hepth/9802109].

3. E. Witten, "Anti-de Sitter space and holography," Adv. Theor. Math. Phys. 2, 253 (1998) [arXiv:hep-th/9802150].

4. O. Aharony, S. S. Gubser, J. M. Maldacena, H. Ooguri and Y. Oz, "Large N field theories, string theory and gravity," Phys. Rept. 323, 183 (2000) [arXiv:hep-th/9905111].

5. G. Policastro, D. T. Son and A. O. Starinets, "The shear viscosity of strongly coupled N = 4 supersymmetric Yang-Mills plasma," Phys. Rev. Lett. 87, 081601 (2001) [arXiv:hep-th/0104066].

6. A. Buchel and J. T. Liu, "Universality of the shear viscosity in supergravity," Phys. Rev. Lett. 93, 090602 (2004) [arXiv:hep-th/0311175].

7. P. Kovtun, D. T. Son and A. O. Starinets, "Viscosity in strongly interacting quantum field theories from black hole physics," Phys. Rev. Lett. 94, 111601 (2005) [arXiv:hep-th/0405231].

8. P. Kovtun, D. T. Son and A. O. Starinets, "Holography and hydrodynamics: Diffusion on stretched horizons," JHEP 0310, 064 (2003) [arXiv:hepth/0309213].

9. K. Maeda, M. Natsuume and T. Okamura, "Viscosity of gauge theory plasma with a chemical potential from AdS/CFT," Phys. Rev. D 73, 066013 (2006) [arXiv:hep-th/0602010].

10. O. Saremi, "The viscosity bound conjecture and hydrodynamics of M2-brane theory at finite chemical potential," JHEP 0610, 083 (2006) [arXiv:hepth/0601159].

11. D. T. Son and A. O. Starinets, "Hydrodynamics of R-charged black holes," JHEP 0603, 052 (2006) [arXiv:hep-th/0601157].

12. J. Mas, "Shear viscosity from R-charged AdS black holes," JHEP 0603, 016 (2006) [arXiv:hep-th/0601144].

13. A. Buchel, R. C. Myers, M. F. Paulos and A. Sinha, "Universal holographic hydrodynamics at finite coupling," Phys. Lett. B 669, 364 (2008) [arXiv:0808.1837 [hep-th]].

14. A. Buchel, "Resolving disagreement for eta/s in a CFT plasma at finite coupling," Nucl. Phys. B 803, 166 (2008) [arXiv:0805.2683 [hep-th]].

15. R. C. Myers, M. F. Paulos and A. Sinha, "Quantum corrections to eta/s," Phys. Rev. D 79, 041901 (2009) [arXiv:0806.2156 [hep-th]].

16. A. Buchel, "Shear viscosity of CFT plasma at finite coupling," Phys. Lett. B 665, 298 (2008) [arXiv:0804.3161 [hep-th]].

17. A. Buchel, "Shear viscosity of boost invariant plasma at finite coupling," Nucl. Phys. B 802, 281 (2008) [arXiv:0801.4421 [hep-th]].

18. A. Buchel, J. T. Liu and A. O. Starinets, "Coupling constant dependence of the shear viscosity in N=4 supersymmetric Yang-Mills theory," Nucl. Phys. B 707, 56 (2005) [arXiv:hep-th/0406264].

19. P. Benincasa and A. Buchel, "Transport properties of $\mathrm{N}=4$ supersymmetric Yang-Mills theory at finite coupling," JHEP 0601, 103 (2006) [arXiv:hep- 
th/0510041].

20. R. G. Cai and Y. W. Sun, "Shear Viscosity from AdS Born-Infeld Black Holes," JHEP 0809, 115 (2008) [arXiv:0807.2377 [hep-th]].

21. M. Brigante, H. Liu, R. C. Myers, S. Shenker and S. Yaida, "Viscosity Bound Violation in Higher Derivative Gravity," Phys. Rev. D 77, 126006 (2008) [arXiv:0712.0805 [hep-th]].

22. R. Brustein, D. Gorbonos and M. Hadad, "Wald's entropy is equal to a quarter of the horizon area in units of the effective gravitational coupling," Phys. Rev. D 79, 044025 (2009) [arXiv:0712.3206 [hep-th]].

23. R. Brustein and A. J. M. Medved, "The ratio of shear viscosity to entropy density in generalized theories of gravity," Phys. Rev. D 79, 021901 (2009) [arXiv:0808.3498 [hep-th]].

24. R. Brustein and A. J. M. Medved, "The shear diffusion coefficient for generalized theories of gravity," Phys. Lett. B 671, 119 (2009) [arXiv:0810.2193 [hep-th]].

25. N. Iqbal and H. Liu, "Universality of the hydrodynamic limit in AdS/CFT and the membrane paradigm," Phys. Rev. D 79, 025023 (2009) [arXiv:0809.3808 [hep-th]].

26. R. G. Cai, Z. Y. Nie and Y. W. Sun, "Shear Viscosity from Effective Couplings of Gravitons," Phys. Rev. D 78, 126007 (2008) [arXiv:0811.1665 [hepth]].

27. G. Policastro, D. T. Son and A. O. Starinets, "From AdS/CFT correspondence to hydrodynamics," JHEP 0209, 043 (2002) [arXiv:hep-th/0205052].

28. D. T. Son and A. O. Starinets, "Viscosity, Black Holes, and Quantum Field Theory," Ann. Rev. Nucl. Part. Sci. 57, 95 (2007) [arXiv:0704.0240 [hep-th]].

29. P. K. Kovtun and A. O. Starinets, "Quasinormal modes and holography," Phys. Rev. D 72, 086009 (2005) [arXiv:hep-th/0506184].

30. M. Brigante, H. Liu, R. C. Myers, S. Shenker and S. Yaida, "The Viscosity Bound and Causality Violation," Phys. Rev. Lett. 100, 191601 (2008) [arXiv:0802.3318 [hep-th]].

31. Y. Kats and P. Petrov, "Effect of curvature squared corrections in AdS on the viscosity of the dual gauge theory," JHEP 0901, 044 (2009) [arXiv:0712.0743 [hep-th]].

32. I. P. Neupane, Int. J. Mod. Phys. A 24, 3584 (2009) [arXiv:0904.4805 [gr-qc]].

33. I. P. Neupane and N. Dadhich, arXiv:0808.1919 [hep-th].

34. S. S. Pal, " $\eta / s$ at finite coupling," arXiv:0910.0101 [hep-th].

35. N. Banerjee and S. Dutta, "Near-Horizon Analysis of $\eta / s$," arXiv:0911.0557 [hep-th].

36. D. W. Pang, "On Charged Lifshitz Black Holes," arXiv:0911.2777 [hep-th].

37. N. Banerjee and S. Dutta, "Higher Derivative Corrections to Shear Viscosity from Graviton's Effective Coupling," JHEP 0903, 116 (2009) [arXiv:0901.3848 [hep-th]].

38. N. Banerjee and S. Dutta, "Shear Viscosity to Entropy Density Ratio in Six Derivative Gravity," JHEP 0907, 024 (2009) [arXiv:0903.3925 [hep-th]].

39. D. W. Pang, " $R^{2}$ Corrections to Asymptotically Lifshitz Spacetimes," JHEP 0910, 031 (2009) [arXiv:0908.1272 [hep-th]]. 
40. M. F. Paulos, "Transport coefficients, membrane couplings and universality at extremality," arXiv:0910.4602 [hep-th].

41. R. G. Cai, Z. Y. Nie, N. Ohta and Y. W. Sun, "Shear Viscosity from GaussBonnet Gravity with a Dilaton Coupling," Phys. Rev. D 79, 066004 (2009) [arXiv:0901.1421 [hep-th]].

42. R. G. Cai, Y. Liu and Y. W. Sun, "Transport Coefficients from Extremal Gauss-Bonnet Black Holes," arXiv:0910.4705 [hep-th].

43. X. H. Ge, Y. Matsuo, F. W. Shu, S. J. Sin and T. Tsukioka, "Viscosity Bound, Causality Violation and Instability with Stringy Correction and Charge," JHEP 0810, 009 (2008) [arXiv:0808.2354 [hep-th]].

44. M. Edalati, J. I. Jottar and R. G. Leigh, "Transport Coefficients at Zero Temperature from Extremal Black Holes," arXiv:0910.0645 [hep-th].

45. T. Faulkner, H. Liu, J. McGreevy and D. Vegh, "Emergent quantum criticality, Fermi surfaces, and AdS2," arXiv:0907.2694 [hep-th].

46. S. J. Rey, "String Theory On Thin Semiconductors: Holographic Realization Of Fermi Points And Surfaces," Prog. Theor. Phys. Suppl. 177, 128 (2009) [arXiv:0911.5295 [hep-th]].

47. S. S. Lee, "A Non-Fermi Liquid from a Charged Black Hole: A Critical Fermi Ball," Phys. Rev. D 79, 086006 (2009) [arXiv:0809.3402 [hep-th]].

48. H. Liu, J. McGreevy and D. Vegh, "Non-Fermi liquids from holography," arXiv:0903.2477 [hep-th]. 